\title{
Lasers without inversion (LWI) in Space: A possible explanation for intense, narrow-band, emissions that dominate the visible and/or far-UV (FUV) spectra of certain astronomical objects
}

\author{
P. P. Sorokin and J. H. Glownia
}

IBM Research Division, PO Box 218, Yorktown Heights, NY 10598, USA

Received 3 December 2001 / Accepted 20 December 2001

\begin{abstract}
The optical or far-UV (FUV) spectra of certain objects in Space are completely dominated by one or two spectrally narrow emission lines, strongly suggesting that laser action of some kind occurs in these objects. However, the electronic level structures of the atoms/ions producing these emissions preclude the possibility of maintaining population inversions on the electronic transitions involved. In lasers, gain is normally produced on an optical transition that is inverted, i.e. one that has more atoms maintained in the upper than in the lower state, so that stimulated emission can exceed stimulated absorption. However, as a result of discoveries made in quantum electronics over the past 30 years or so, one now knows that there are several ways to make stimulated emission occur on a transition that is not inverted, i.e. to realize a "laser without inversion (LWI)". This requires first making the atoms non-absorbing at the lasing frequency, i.e. setting up a condition of "electromagnetically induced transparency (EIT)". Some recently developed EIT techniques for three-level atoms are first reviewed. A simple model for a space LWI based upon a gas of two-level atoms is then proposed. In this model, transparency results from a form of EIT induced by the presence of an intense, monochromatic, continuous-wave, laser beam tuned to the frequency $\omega_{\mathrm{o}}$ of the two-level-atom transition. Amplification of light at this same frequency occurs via resonant stimulated hyper-Raman scattering (SHRS) and four-wave mixing (FWM), with pumping energy provided by continuum starlight spectrally overlapping the two outer absorption sidebands ("Mollow bands") induced by the presence of the beam at $\omega_{\mathrm{o}}$. Two specific examples of superintense line emission from Space are here considered. These are (a) the $\mathrm{H}(\alpha)$ emission line appearing as a dominant singularity in certain reddened, early-type stars, and (b) the powerful O VI (1032 $\AA, 1038 \AA)$ emission doublet that dominates the FUV emission spectra of symbiotic stars such as RR Tel.
\end{abstract}

Key words. atomic processes - radiation mechanisms: non-thermal - stars: early type - stars: individual: HD 44179 - stars: individual: RR Tel - ISM: lines and bands

\section{Introduction}

The introduction of an entirely new concept to explain a class of observed phenomena in any field of science would seem to be a redundant activity, if the phenomena can reasonably be accounted for with use of existing theories and models. However, in the cases of certain astronomical observations of anomalously intense emission lines, standard explanations appear to be inadequate. This paper focuses specifically on the $\mathrm{H}(\alpha)$ emission line appearing as an intense, $\delta$-function-like, singularity in the spectra of some reddened early-type stars, and also on the powerful O VI (1032 A, $1038 \AA$ ) emission doublet that dominates the FUV spectra of symbiotic stars such as RR Tel. The fact that one or two sharp spectral lines can dominate the total emission spectrum of any space object over a very wide

Send offprint requests to: P. P. Sorokin,

e-mail: sorokin@us.ibm.com spectral range suggests that laser action of some kind is occurring in that object. However, as is shown below, under typical conditions existing in astrophysical environments surrounding bright stars, the electronic level structures of both $\mathrm{H}$ atoms and $\mathrm{O}$ VI ions preclude the possibility of population inversions occurring on any of the transitions producing the intense emissions that are observed. Since population inversion is generally thought to be a prerequisite for lasing, this would appear to rule out immediately any possibility that the latter could be the cause of the observed anomalously strong emission lines. However, as a result of theoretical and experimental studies done over the past 30 years, one now knows that there are ways in which laser action can occur on a transition without a population inversion being present on that transition. This fact is the essential message conveyed by many recent studies performed in the relatively new research area of quantum electronics termed "lasers without inversion 
(LWI)". The first step in realizing an LWI is to cancel somehow the absorption arising from the noninverted atoms. This can be accomplished through a variety of recently developed techniques based upon quantum interference that create a condition in the LWI medium known as "electromagnetically induced transparency (EIT)". Part of the present paper is devoted to a brief review of some especially striking results achieved in the EIT field.

In Sect. 2, a few spectra taken from a recent astronomical survey are displayed which strikingly demonstrate that enormously enhanced $\mathrm{H}(\alpha)$ emissions are indeed seen in some reddened, early-type stars. Yet from a portion of the $\mathrm{H}$-atom energy level diagram that includes the upper and lower levels of the $\mathrm{H}(\alpha)$ transition, one readily sees that it would be virtually impossible to have a population inversion continuously maintained on this same transition. In Sect. 3, spectra of three symbiotic stars are presented to illustrate just how dominant the O VI (1032 A, $1038 \AA)$ emission doublet can be in the FUV spectra of such stars. In this case, maintaining population inversions on the two emitting transitions is made even more difficult by virtue of the fact that the lower level of each transition is the O VI ion ground state level. Presaging further the possible necessity for a new paradigm to be here considered are serious difficulties one encounters when trying to account for symbiotic star O VI doublet emission through conventional means. For example, linear optical pumping of O VI ions by FUV continuum light from the hot white dwarf member can only result in elastic scattering of the light. No real excitation of the two upper O VI levels can be produced in this manner in an atmosphere as rarified as that characterizing the gas occupying the region between the two members of a symbiotic star. Again, if one assumes that excitation of the O VI upper levels occurs via recombination of ions and electrons, one is then faced with the difficulty of explaining why the intensity ratio of the two emission components of the O VI doublet is observed to vary greatly from the theoretically expected $2: 1$ ratio in the various symbiotic star systems surveyed.

Sensing that a radically new approach might indeed be needed to explain the superintense emission lines from Space described above, in Sect. 4 we consider some of the striking results that have been achieved in the fields of EIT and coherently phased atoms. Most recent research in these areas has focussed on three-level atomic structures, which allow two nonlinearly-coupled resonant light waves to propagate together through a medium without being at all attenuated. It seems reasonable to speculate that this finding might form a cornerstone for a space laser model that could explain intense $\mathrm{H}(\alpha)$ or O VI emissions from Space. Less apparent is the likelihood that another striking discovery made by investigators in the EIT field ultraslow propagation of light - can also play an important role in determining the amount of gain (i.e. optical amplification per unit propagation distance) one calculates for a space laser model in which the signal wave group velocity depends upon EIT. An attempt to find an answer to this question is nonetheless made in Sect. 4.
The exact nature of the pumping mechanism is often only vaguely specified in the various schemes for LWI that have been considered in the literature. In proposing that a form of LWI occurs around certain objects in Space, we initially consider a somewhat unusual nonlinear pumping mechanism termed stimulated hyper-Raman scattering. This involves multi-photon absorption of continuum light from a nearby illuminating star via new resonances that are induced in two-level atoms when intense, monochromatic light is applied at the transition frequency. The transparency required for a two-level-atom LWI to operate results both from the effect of saturation and from a form of EIT that occurs in two-level-atom gases. This leads to a greatly simplified space laser model, as is explained in Sect. 5. The optical gain of a 2-level LWI operating in a symbiotic star environment is calculated in Sect. 5, and is found to be more than sufficient, implying rather strongly that LWI's must indeed be the generators of the superintense (1032 $\AA, 1038 \AA$ ) O VI doublet emissions seen in these space objects. It is also concluded in Sect. 5 that 2-level lasing without inversion is the most likely explanation of superintense $\mathrm{H}(\alpha)$ emissions seen in some reddened, early-type stars. However, lack of detailed knowledge about the astrophysical environments of these objects makes this conclusion somewhat less certain.

\section{2. $\mathrm{H}(\alpha)$ emissions in reddened early-type stars}

In Rawlings et al. (2000) new optical spectroscopy for $45 \%$ of an older catalog (Stephenson 1992) of $\sim 440$ reddened stars was performed in an attempt to isolate reddened stars which are also early-type. The spectra between $6100 \AA$ and $6900 \AA$ of all the stars studied are shown in Rawlings et al. (2000). Only fifteen of the stars surveyed could be classified as early-type stars. With regard to $\mathrm{H}(\alpha)$, the spectra of these early-type stars show tremendous variations. Some (Fig. 1a) show $\mathrm{H}(\alpha)$ only in absorption. Others (Figs. 1b-d) show $\mathrm{H}(\alpha)$ strongly in emission, with an intensity completely disproportionate to all other absorption features present. Yet the spectral types of these stars are all apparently roughly comparable. In the spectra of all the cooler stars surveyed by Rawlings et al. (2000), $\mathrm{H}(\alpha)$, if present at all, appears only in absorption.

Much spectral data exists for the star, HD 44179, that powers the Red Rectangle nebula. We here consider an apertured line-of-sight that includes just the star, not the nebula. In such a line-of-sight, one sees (Cohen et al. 1975) that all Balmer lines, with the notable exception of $\mathrm{H}(\alpha)$, appear as broad absorptions against the star's blackbody continuum. No emissions are present in the blue-green spectral region. However, $\mathrm{H}(\alpha)$ appears as a strong, narrow, emission line centrally placed in a broad absorption. A recent STIS scan showing $\mathrm{H}(\alpha)$ emission in HD 44179 is shown in Fig. 2.

In view of the enormous variations in $\mathrm{H}(\alpha)$ spectral width and intensity observed in lines-of-sight to reddened stars of roughly the same spectral type, a non-stellar origin for this emission is strongly suggested. Hence - at 

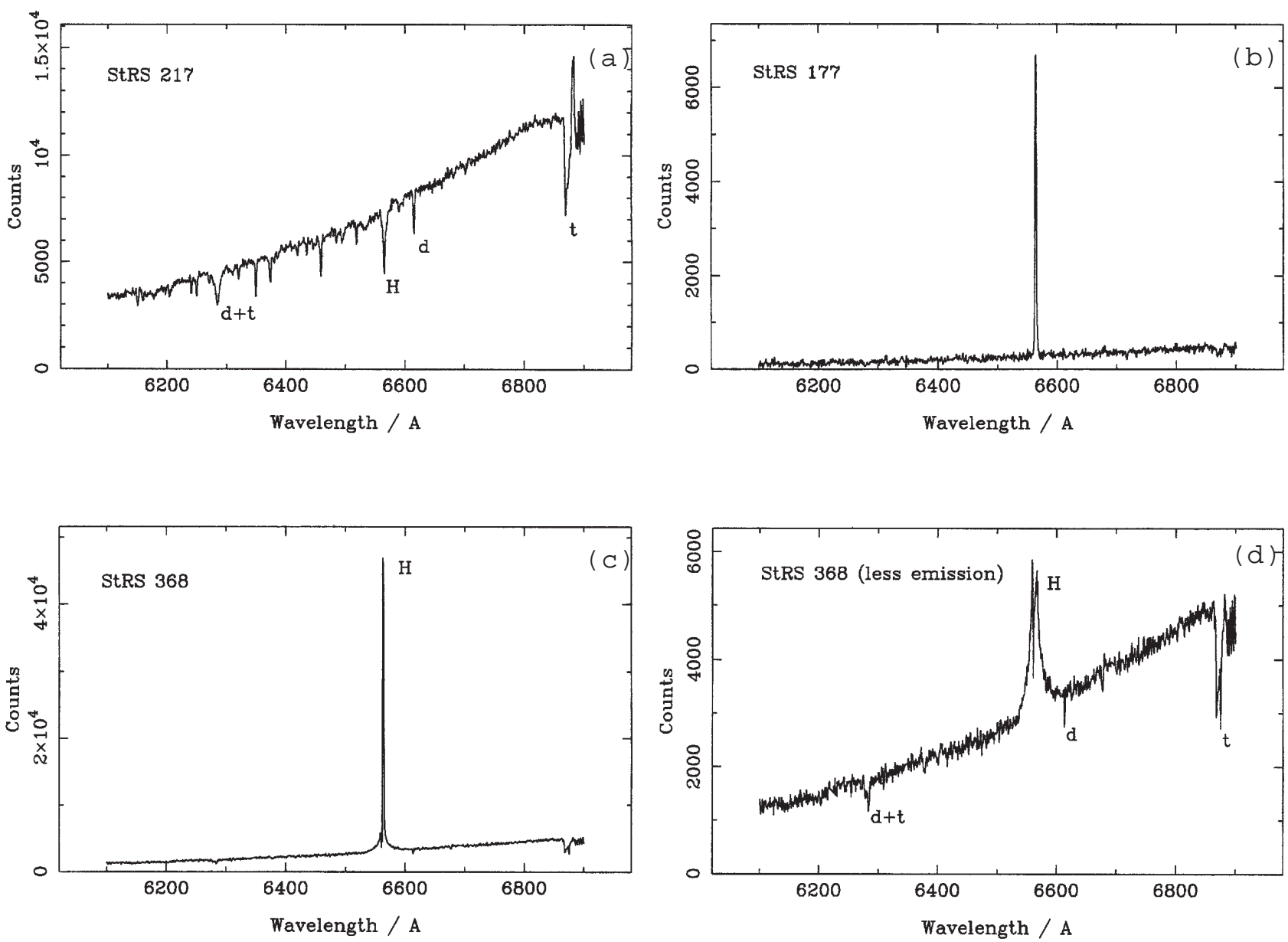

Fig. 1. a-d) Spectra of three reddened early-type stars selected from Fig. 4 of Rawlings et al. (2000). Features labeled "d" and " $t$ " are diffuse interstellar and telluric features, respectively. Stars labeled by running numbers used in Table 1 of Stephenson (1992). (StRS $\equiv$ "Stephenson Reddened Star".) Standard identifications for StRS 177 and StRS 368 are IRAS $18179-1346$ and IRAS 20298+4011, respectively.

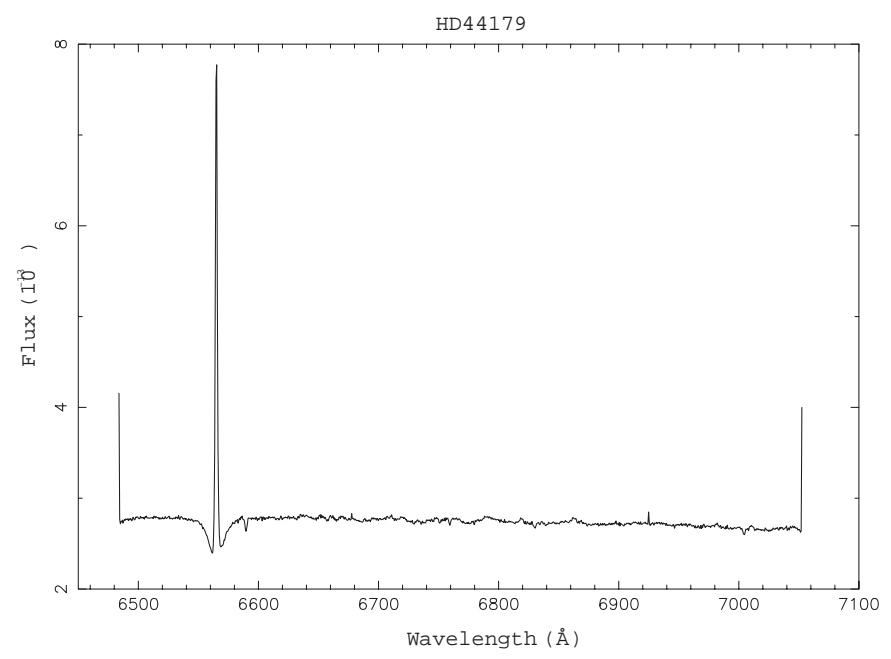

Fig. 2. STIS scan of HD 44179 taken on Mar 26, 1998 (T. Gull, principal investigator). Spectrum obtained from HST web archive.

least in the most singular cases - this emission must originate from $\mathrm{H}$ atoms located outside the photosphere of the illuminating star. If the $\mathrm{H}(\alpha)$ emissions resulted from electron impact excitation occurring in compact $\mathrm{H}$ II regions about the stars, one would expect also to detect emissions at the wavelength of $\mathrm{H}(\beta), \mathrm{H}(\gamma), \mathrm{H}(\delta)$, etc. At least in the case of HD 44179, these are not observed.

At first glance, one would also think that the $\mathrm{H}(\alpha)$ emissions could reasonably be produced via linear photoexcitation of $\mathrm{H}$ atoms located in neutral regions bordering thin H II regions. A portion of the $\mathrm{H}$-atom energy level diagram that contains both upper and lower levels of the $\mathrm{H}(\alpha)$ transition is shown in Fig. 3. Transitions $1 \Longleftrightarrow 3$ and $2 \Longleftrightarrow 3$ are allowed; the total radiative decay rate of quantum level $|3\rangle$ is $\sim 10^{9} \mathrm{~s}^{-1}$, with most of the fluorescent emission occurring on the transition $2 \Longleftrightarrow 3$. Since $|2\rangle$ decays radiatively to $|1\rangle$ via spontaneous two-photon emission at a rate $\approx 8 \mathrm{~s}^{-1}$, transition $1 \Longleftrightarrow 2$ is basically forbidden. A typical $\mathrm{H}$-atom concentration existing in a cold neutral cloud surrounding an early-type star might be $\sim 10^{4} \mathrm{~cm}^{-3}$. The corresponding collision rate would be $\sim 10^{-7}-10^{-8} \mathrm{~s}^{-1}$ - low enough for the gaseous medium to be realistically considered collisionless. Weisskopf (1931) first showed that if a two-level atom in a collisionless medium is excited by weak, monochromatic, resonant 


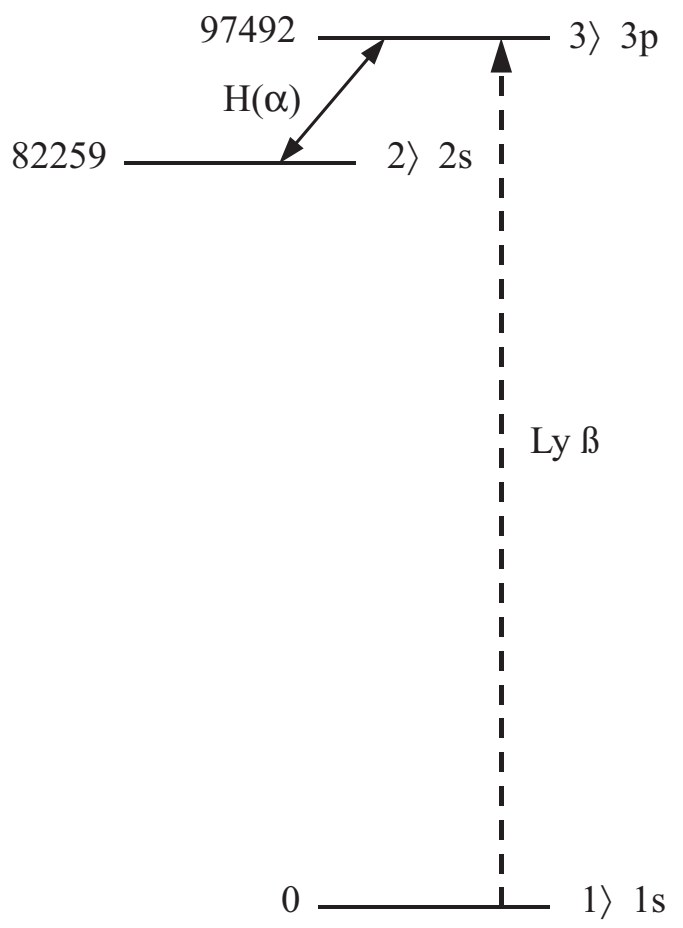

Fig. 3. Partial energy level diagram for $\mathrm{H}$ atoms, showing the levels involved in $\mathrm{H}(\alpha)$ emission from stars.

radiation, the resulting fluorescence is also monochromatic and has the same frequency as the incident light. In other words, the scattering is purely elastic, and no real excitation of the upper level occurs. This statement ceases to hold for the case of strong field excitation, when the induced transition rate is comparable to, or greater than, the spontaneous emission rate. It was, of course, the advent of the laser that stimulated theoreticians in quantum electronics to work out eventually the correct expressions for both (a) the absorption spectrum (Mollow 1972) and (b) the fluorescence spectrum (Mollow 1969) of a two-level atom excited by an intense, monochromatic, field. When the exciting monochromatic field is exactly on resonance, there is a range of power levels for which both spectral distributions consist largely of three peaks. This same range of power levels will concern us in Sect. 5 , and we will there refer to the three peaks as "Mollow bands".

The " $\Lambda$ " system shown in Fig. 3 is a three-level system, not a two-level system. Under collisionless conditions, when this system is excited by weak, monochromatic excitation anywhere in a narrow spectral range spanning the $1 \Longleftrightarrow 3$ transition, both elastic scattering and spontaneous Raman scattering occur (Loudon 2000), but, again, as in the two-level case, no real excitation of quantum level $|3\rangle$ takes place. The light produced by the Raman scattering process would have a broadened spectral profile centered on $\mathrm{H}(\alpha)$, but it would be radiated into $4 \pi$ steradians and cannot therefore account for the extreme $\delta$-function-like profiles observed in, for example, Figs. $1 \mathrm{~b}$ and $1 \mathrm{c}$.

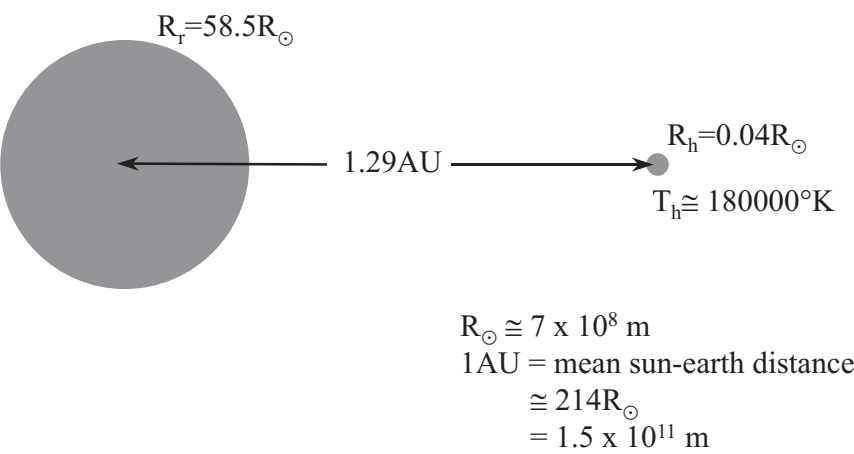

RW Hydrae

Fig. 4. Sketch of the geometry of the symbiotic star RW Hydrae, based upon measurements presented in Schild et al. (1996).

The occurrence of stimulated emission or lasing on the $2 \Longleftrightarrow 3$ transition could easily account for $\mathrm{H}(\alpha)$ emissions from Space that appear sharp, intense, and singularlooking (i.e. unaccompanied by a significant pedestal). However, it is a trivial matter to show that it would be virtually impossible to have a population inversion continuously maintained on the $2 \Longleftrightarrow 3$ transition. From Fig. 3, one sees that in the steady state the following condition must hold:

$n_{3} \Gamma_{32}=n_{2} \Gamma_{21}$,

where the $n$ 's are the level population densities and the $\Gamma$ 's are (one half) the radiative decay rates. (For a lifetime broadened transition, $2 \Gamma$ is the FWHM linewidth of the Lorentzian profile expressed in $\operatorname{rad~s}^{-1} \cdot 2 \Gamma=A=1 / \tau$, where $A$ is the Einstein coefficient and $\tau$ is the radiative lifetime.) Since $\Gamma_{32} \gg \Gamma_{21}$, no population inversion can continually be maintained on the $2 \Longleftrightarrow 3$ transition.

\section{The 0 VI (1032 Å, $1038 \AA ̊)$ emission doublet in symbiotic stars}

A symbiotic star is generally considered by astronomers to consist of two stars orbiting together in Space while remaining a fixed distance apart from one another. One of the stars is assumed to be a decaying red giant, the other an extremely hot white dwarf. The red giant continually loses mass; some of this mass accretes onto the white dwarf, leading to additional heating of the latter. A typical geometry for a symbiotic star is shown in Fig. 4.

Much of the enthusiasm that currently exists for symbiotic star research was stimulated by a series of recent spectral studies. Symbiotic stars often show two emission lines at $6825 \AA$ and $7082 \AA$. The origin of these lines remained for many years a mystery in astronomy, until they were identified as spontaneous Raman scattering of the O VI (1032 A, $1038 \AA)$ resonance doublet by hydrogen atoms (Schmid 1989). These spontaneous Raman scattering processes are shown in Fig. 5. For the past ten years or so, these lines have provided astronomers with a new 


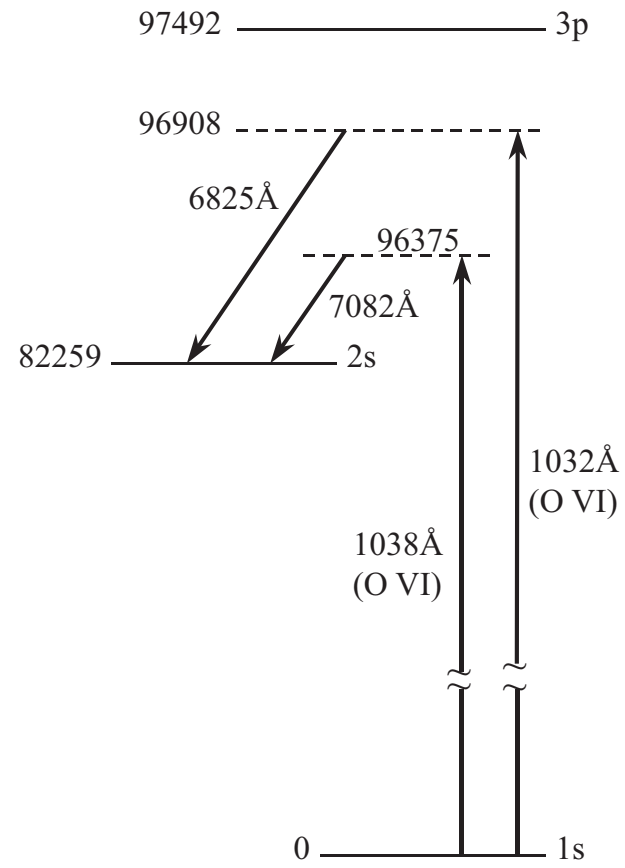

Fig. 5. Spontaneous Raman scattering processes responsible for producing emissions observed at $6825 \AA$ and $7082 \AA$ in symbiotic stars.

and powerful diagnostic tool for determining the geometric structure of symbiotic systems. Spectropolarimetric observations reveal that the Raman lines are polarized. In symbiotic stars the $\mathrm{O}$ VI region is assumed to be close to the hot component, and the H-atom concentration is known to be large near the red giant. Thus the polarization angle provides the orientation of the binary axis of the symbiotic system.

Although measurements of the red lines alone could provide all the information needed for the structures of symbiotic star systems to be determined, it was only comparatively recently that high quality FUV spectra of some of these objects were recorded (Schmid et al. 1999). The O VI lines of several symbiotic stars were observed during the ORFEUS - SPAS I and ORFEUS - SPAS II space shuttle missions STS-51 (September 1993) and STS80 (November/December 1996). The ORFEUS telescope fed two instruments: the Berkeley spectrometer with spectral resolution $\sim 3000$ and the Echelle spectrometer with spectral resolution $\sim 10000$. Details of the experimental techniques employed and examples of spectra taken with the Berkeley spectrometer on both missions and with the Echelle spectrometer during the second mission are presented in Schmid et al. (1999). In Figs. 6-8 are shown Berkeley spectra of three symbiotic stars recorded during the first space shuttle mission.

One sees from these spectra that the O VI doublet emission indeed completely dominates the FUV spectra of these symbiotic stars over a wide frequency range. Again, one senses that a lasing process of some kind must be involved in order for such relatively intense, narrow-band, emissions to be produced. Yet on the basis of an O VI

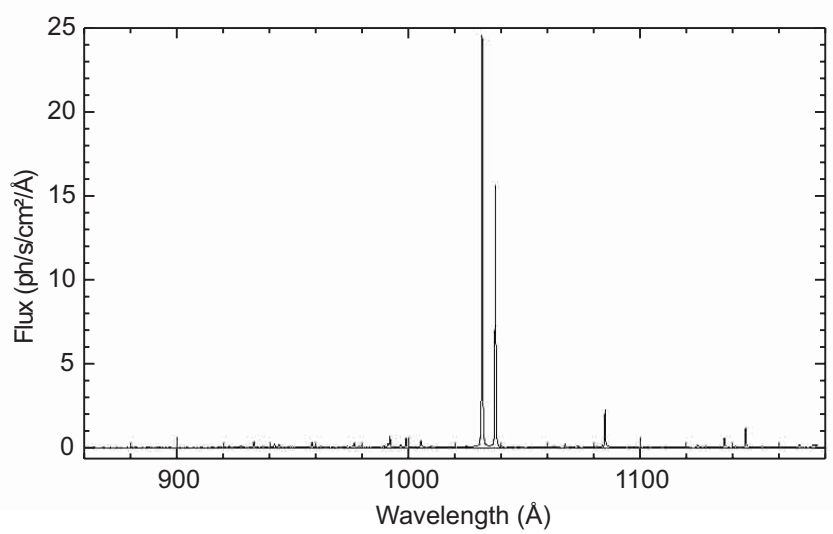

Fig. 6. Spectrum of RR Tel from ORFEUS I spectral browser. http://albert.ssl.berkeley.edu/orfeus/ spec-list-rr_tel.html

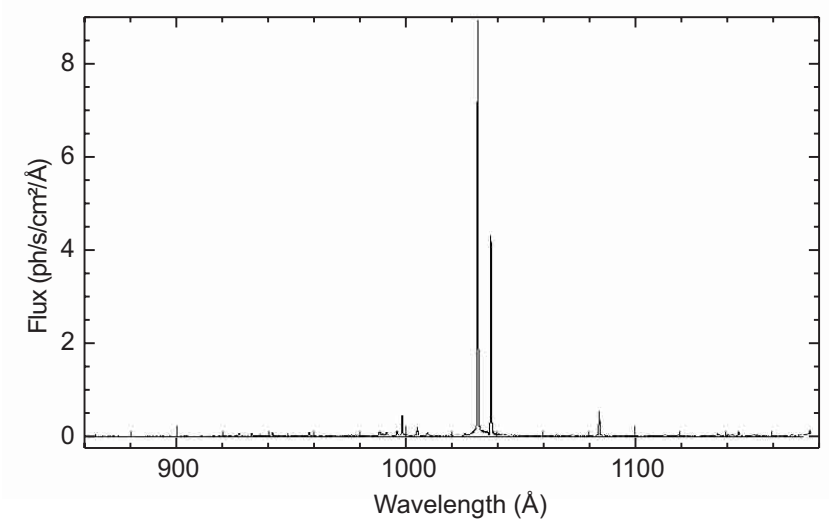

Fig. 7. Spectrum of AG Dra from ORFEUS I spectral browser. http://albert.ssl.berkeley.edu/orfeus/ spec-list-ag_dra.html

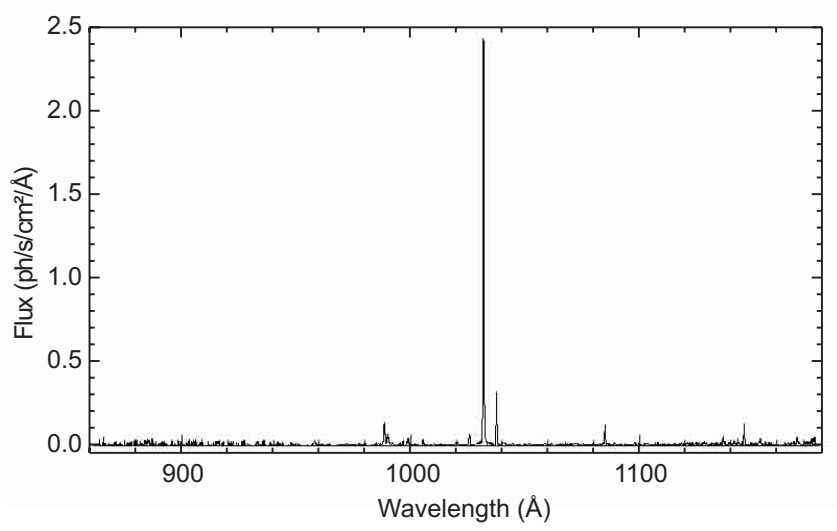

Fig. 8. Spectrum of $\mathrm{Z}$ And from ORFEUS I spectral browser. http://albert.ssl.berkeley.edu/orfeus/ spec-list-z_and.html

energy level diagram (Fig. 9) that includes the transitions on which the light is emitted, it appears very difficult to provide a reasonable scenario showing just how intense, narrow-band, light emission is generated in symbiotic stars. It is perhaps even more difficult to do this than 


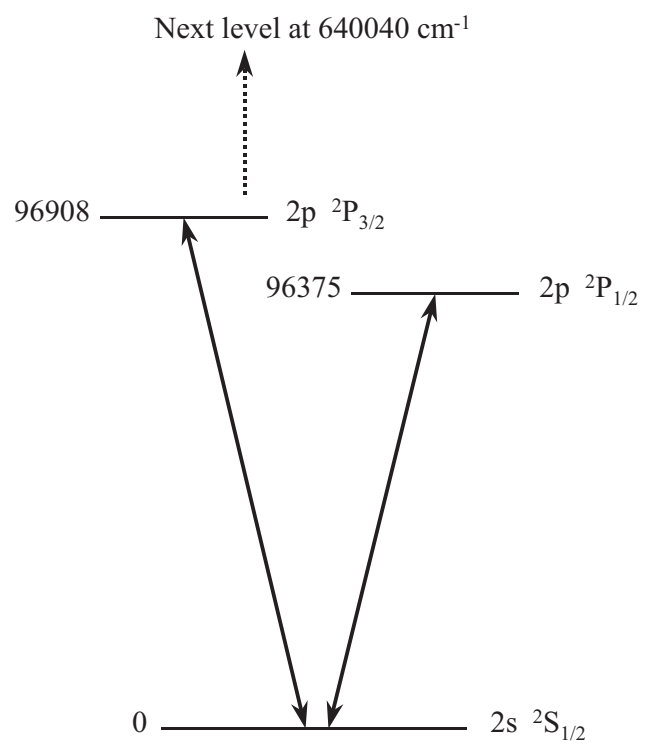

Fig. 9. Energy level diagram for the O VI ion.

it was to try to explain the intense, narrow-band, $\mathrm{H}(\alpha)$ emissions observed in some reddened, early-type stars. For one thing, to maintain population inversions on both transitions of the (1032 $\AA, 1038 \AA$ ) doublet would require that at least two-thirds of all the O VI ions must at any time reside in the excited state levels. This would require the continuous application of unattainable high pump powers. Linear pumping of the excited state levels by the FUV continuum radiated by the hot white dwarf can result only in elastic scattering, not real excitation of the levels, and cannot therefore explain the emissions. Regarding possible excitation of the levels through either electron impact or recombination of ions and electrons, there is a fairly strong indication in Figs. 6-8 that this is not what happens in symbiotic stars. Excitation by electronic impact or via ionelectron recombination should theoretically always result in a 2:1 intensity ratio for the fluxes from the two doublet components. The (1032 A, $1038 \AA$ ) line fluxes determined from the Berkeley spectra are given in Table 2 of Schmid et al. (1999). The line flux ratios vary from $\sim 10$ ( $\mathrm{Z}$ And) to $\sim 1.3$ (RR Tel).

One here might anticipate examining superintense symbiotic star O VI emission in the light of LWI/EIT concepts by noting that the three levels of O VI shown in Fig. 9 would form a particularly "clean" example of a "V"-type three-level system that is frequently considered in the context of LWI/EIT. Firstly, the next highest level of O VI occurs at $640040 \mathrm{~cm}^{-1}$. The three levels shown in Fig. 9 therefore form a closed system, with no effective radiative or non-radiative couplings to any other levels. Secondly, the even mass number $\mathrm{O}^{16}$ isotope $(99.76 \%$ abundant) has no nuclear spin. Therefore, there is no hyperfine splitting in any of the O VI electronic levels. In atoms such as $\mathrm{Na}^{23}$, hyperfine splitting can greatly complicate LWI/EIT experiments.

\section{EIT in three-level atomic systems}

The majority of EIT studies are concerned with three-level atom systems, which for the most part are either " $\Lambda$ "-type, as in Fig. 3, or "V"-type, as in Fig. 9. In one approach, pioneered by the group of S. E. Harris, the emphasis has generally been to consider how a weak monochromatic "probe beam", applied in the vicinity of the $\omega_{13}$ transition in a $\Lambda$-type three-level system, propagates in a gas of such atoms when a strong monochromatic laser beam (the EIT "coupling beam"), tuned to line center $\omega_{23}$ of the $2 \Longleftrightarrow 3$ transition, copropagates in the same gas. In another approach, copropagation of probe and coupling beams is studied when there is no constraint that the intensity of the probe beam be small compared to that of the coupling beam. At first glance, this second approach would appear to be of more relevance for a space laser model. However, since valuable insight is likely to be gained by examining results obtained in the first approach, we now proceed to do so.

Let us specifically consider a $\Lambda$-type three-level atom having an energy level diagram similar to that in Fig. 3. Transitions $1 \Longleftrightarrow 3$ and $2 \Longleftrightarrow 3$ are allowed. Transition $1 \Longleftrightarrow 2$ is assumed to be basically forbidden. (One half) the total radiative decay rate of quantum level $|3\rangle$ is $\Gamma_{3}$, i.e. $\Gamma_{3}=\Gamma_{31}+\Gamma_{32}$. The quantity $\Gamma_{21}$, which is assumed to be nearly zero, is for simplicity here designated by $\Gamma_{2}$.

When a strong monochromatic laser beam (the EIT "coupling beam"), tuned to line center $\omega_{23}$ of the $2 \Longleftrightarrow 3$ transition, is applied to a gas of three-level atoms, a sharp dip in absorption in the vicinity of the $1 \Longleftrightarrow 3$ transition occurs (Fig. 10a). This cancellation in the imaginary part of the linear susceptibility (absorption is proportional to Im $\chi^{(1)}$ ) can be shown to arise from quantum interference. For the ideal case $\Gamma_{2}=0$, there is perfect transparency at the minimum. The width of the transparency hole varies as $\Omega_{23}\left(\equiv \Omega_{\mathrm{c}}\right.$ ), the so-called Rabi frequency of the coupling laser, which is proportional to the square root of the laser power $\left(\Omega_{\mathrm{c}}=\mu_{23} E_{\mathrm{c}} / \hbar\right.$ where $\mu_{23}$ is the transition dipole matrix element and $E_{\mathrm{c}}$ is the optical field of the coupling laser beam). Thus, by applying to a gas of $\Lambda$-type threelevel atoms an intense, monochromatic, laser beam tuned to $\omega_{23}$, one can induce almost perfect transparency in the gas in a narrow spectral interval about $\omega_{13}$. This is a dramatic prediction of EIT which was first demonstrated in the laboratory by S. E. Harris and co-workers (Kasapi et al. 1995). In Harris et al. (1992), formulae characterizing this induced transparency are derived and tabulated. For example, the per length $E$ field loss $\alpha_{E}$ at exact probe resonance is shown to be given by the following formula:

$\alpha_{E}=\left(\frac{\pi}{\lambda}\right)\left(\frac{2 \Gamma_{2}}{\Omega_{\mathrm{c}}^{2}+\Gamma_{2} \Gamma_{3}}\right)\left(\frac{\left|\mu_{13}\right|^{2} N}{\varepsilon_{\mathrm{o}} \hbar}\right)$,

where $N$ is the atom density and $\varepsilon_{\mathrm{o}}$ is the permittivity of free space. Equation (2) shows that perfect transparency results when $\Gamma_{2}=0$.

Figure 10b shows that the real part of the susceptibility (refractive index) exhibits a steep slope with no inflection 

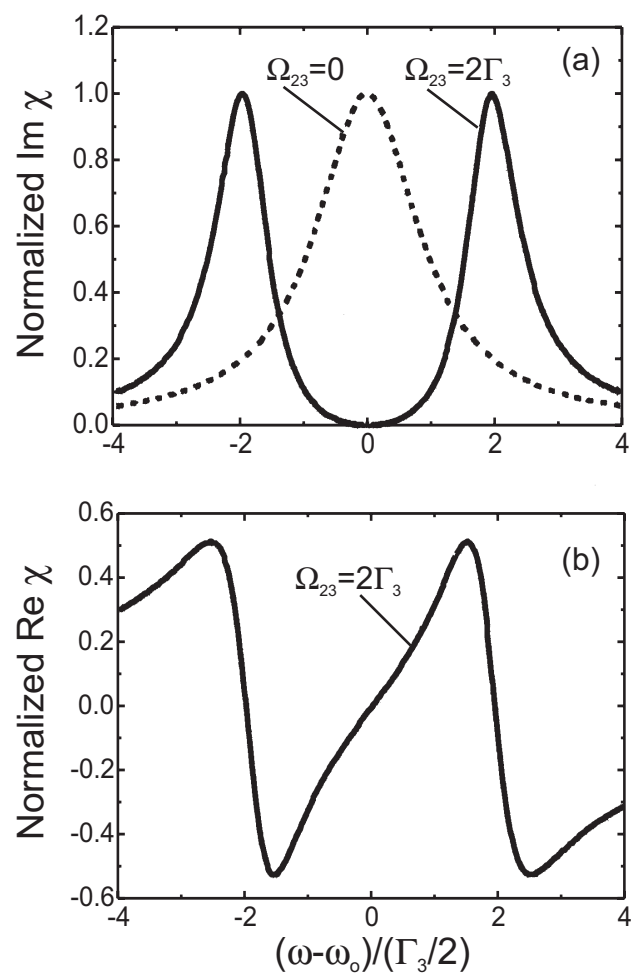

Fig. 10. Imaginary a) and real b) parts of the linear susceptibility for a weak probe pulse of frequency $\omega$ in the presence (solid lines) or absence (dashed line) of a strong coupling field tuned to the transition $\omega_{23}$ of a $\Lambda$-type, three-level atom. Normalization is to the peak value of the imaginary part of the susceptibility. After Fig. 2 of Harris et al. (1990).

point at $\omega=\omega_{13}$. The probe pulse, when tuned exactly to resonance, thus experiences a linear rapidly varying refractive index with very slow group velocity and zero group-velocity dispersion. The latter feature allows resonant pulses to propagate long distances without pulse reshaping. Harris et al. (1992) also present formulas for the dispersive properties of a weak probe pulse propagating in an EIT medium. For example, when the group velocity $v_{\mathrm{g}}$ is significantly slower than $c$, the velocity of light, and $\Gamma_{2}=0$, one has:

$v_{\mathrm{g}} \simeq\left(\frac{\hbar c \varepsilon_{\mathrm{o}}}{2 \omega_{13}}\right)\left(\frac{\left|\Omega_{\mathrm{c}}\right|^{2}}{\left|\mu_{13}\right|^{2} N}\right)$.

With this result, it is possible to estimate how low a group velocity a weak optical light pulse traveling in space could have when its propagation is mediated by the presence of an EIT coupling beam. For simplicity, assume that a $100 \mathrm{~mW} / \mathrm{cm}^{2}$ continuous-wave coupling laser beam is propagating through a tenuous (density $=10^{4} \mathrm{~cm}^{-3}$ ) gas of $\Lambda$-type three-level atoms. Assume the $2 \Longleftrightarrow 3$ transition has a spontaneous emission decay rate $6 \times 10^{8} \mathrm{~s}^{-1}$ and occurs at $6000 \AA$. Thus $\Gamma_{32}=3 \times 10^{8} \mathrm{rad} \mathrm{s}^{-1}$. Assume the transition to which the co-propagating weak probe pulse is tuned decays in a nanosecond and occurs at $1000 \AA$.

The maximum value of the oscillating electric field $E_{\mathrm{c}}$ of the coupling laser beam is $868 \mathrm{~V} / \mathrm{m}$. To find the corresponding Rabi frequency $\Omega_{\mathrm{c}}$, one needs to know the value of $\mu_{23}$. The latter is related to $\Gamma_{32}$ via the general formula (Loudon 2000) given in Eq. (4) below. (This formula assumes equal degeneracy of levels $|2\rangle$ and $|3\rangle$.)

$\Gamma=\frac{\omega^{3}|\mu|^{2}}{6 \varepsilon_{\mathrm{o}} \pi \hbar c^{3}}$

From Eq. (4) and the equation discussed earlier relating $\Omega_{\mathrm{c}}$ to $\left|\mu_{23}\right|$ and $E_{\mathrm{c}}$, one finds that $\Omega_{\mathrm{c}}$ is here $\simeq 5.6 \times 10^{8} \mathrm{rad} \mathrm{s}^{-1}$, corresponding to a frequency of about $89 \mathrm{MHz}$. One can combine Eqs. (3) and (4) to give the following equation for the group velocity of the probe pulse propagating in the EIT medium:

$v_{\mathrm{g}} \simeq \frac{\left|\Omega_{\mathrm{c}}\right|^{2} \omega_{13}^{2}}{12 \pi c^{2} \Gamma_{31} N}$

We thus calculate for our hypothetical case that $v_{\mathrm{g}} \simeq 6.6 \times$ $10^{12} \mathrm{~m} / \mathrm{s}$. This value is greater than $c$, which simply means that use of Eq. (3) here is invalid because $v_{\mathrm{g}}$ is not significantly less than $c$. The density of atoms in space is thus simply too low for slow group velocities to result, even though the medium is made fully transparent at the probe frequency via EIT. Note, however, that if $N$ were $10^{14} \mathrm{~cm}^{-3}, v_{\mathrm{g}}$ would be $\simeq 660 \mathrm{~m} / \mathrm{s}$. In a recent laboratory experiment (Hau et al. 1999), a light speed reduction to $17 \mathrm{~m} / \mathrm{s}$ was achieved in an ultracold atomic gas. Refining the techniques used in this experiment, two groups reported actually "stopping" light in the laboratory (Liu et al. 2001; Phillips et al. 2001).

We now consider the case when the intensity of the monochromatic probe beam is no longer small compared to that of the coupling beam. It is assumed that both beams are here still tuned to the respective line centers of $\Omega_{13}$ and $\Omega_{23}$. What happens in this case is that the atoms in the gas will quickly adjust so that there is also no absorption about the line center of the $2 \Longleftrightarrow 3$ transition. This occurs independently of the ratio of Rabi frequencies $\Omega_{13} / \Omega_{23}$. The two laser beams rapidly drive all atoms of the system into a stable "coherently trapped population state". In this state, the wavefunction of all the atoms in the gas becomes an antisymmetric linear combination of the unperturbed wavefunctions of levels $|1\rangle$ and $|2\rangle$. In this state, no atoms are present in level $|3\rangle$. Atoms coherently trapped by this method are also commonly referred to as "coherently phased" atoms. One of the earliest experiments in which coherent trapping of atomic populations was realized is described in Gray et al. (1978). Coherent trapping can be vividly demonstrated in the laboratory as a complete quenching of fluorescent emission from level $|3\rangle$ when both coupling and probe beams are applied to a vapor cell, whereas when only one or the other of the beams is applied, bright fluorescence is seen. For this reason, the coherently trapped state is sometimes termed a "dark state". Once all the atoms of a gas have been coherently phased by this method, light at the two applied laser frequencies can continue to pass over the atoms without being attenuated. Since there is no effective dipole interaction of either pump or probe beam with the atoms of a 
coherently phased medium, no slower-than-c group velocities are here possible. Although we have continually assumed throughout the discussion presented in this section that coupling and probe beams are tuned exactly to $\omega_{23}$ and $\omega_{13}$, respectively, this is actually not required for the preparation of coherently phased atoms. The frequencies of both beams can each be detuned from the line centers of $\omega_{23}$ and $\omega_{13}$, as long as they remain in exact two-photon resonance with levels $|1\rangle$ and $|2\rangle$.

Although we have up to now focussed in this section on EIT and coherent phasing of $\Lambda$-type three-level atoms, the techniques are known to work as well for V-type threelevel atoms. (In the LWI/EIT literature, a third variety of atomic level structure - the so-called "cascade"-type three-level atom - is also sometimes considered. However, this does not appear to be relevant for our space laser model and (except in Fig. 11) is not discussed in the present paper). Figure 11 shows results from a recent theoretical study of the effects of mismatching wavelengths for the coupling and probe fields in Doppler-broadened media for the three atomic configurations commonly utilized in EIT. Note that, for matched wavelength pulses, EIT seems easiest to excite in the V-type system.

In this section it has been outlined that preparation of coherently phased atoms via co-propagation of two resonantly tuned laser beams through a tenuous gas of threelevel atoms accomplishes one major step needed to realize an LWI in space - it removes in principle all attenuation (loss) for the two beams as they propagate away from the stars that provide the pumping power required for amplification of the beams. In the next section, a specific pumping mechanism for a 2-level LWI is proposed. It will also be shown that there exists a form of EIT for 2-levelatom gases that can provide the transparency required for lasing without inversion in these systems.

\section{A proposed pumping mechanism for an LWI in symbiotic stars: Stimulated hyper-Raman scattering (SHRS) and four-wave mixing (FWM) on transitions between ac-Stark-shifted levels of irradiated 2-level atoms}

Harris et al. (1990) were the first to show that, while EIT makes a material system transparent to resonant laser radiation, it nonetheless allows a large nonlinear resonant process to occur with high probability. On the basis of this general and important finding, we now propose that one such nonlinear resonant process, stimulated hyperRaman scattering (SHRS), might be a viable mechanism for pumping an LWI in symbiotic stars, thus accounting for the superintense (1032 $\AA, 1038 \AA$ ) O VI doublet emission that is seen in such space objects. At the end of this section we will briefly speculate on what might be happening when superintense $\mathrm{H}(\alpha)$ emission is seen in reddened, early-type stars.

Despite the emphasis that has thus far been placed in the present paper on EIT occurring with three-level
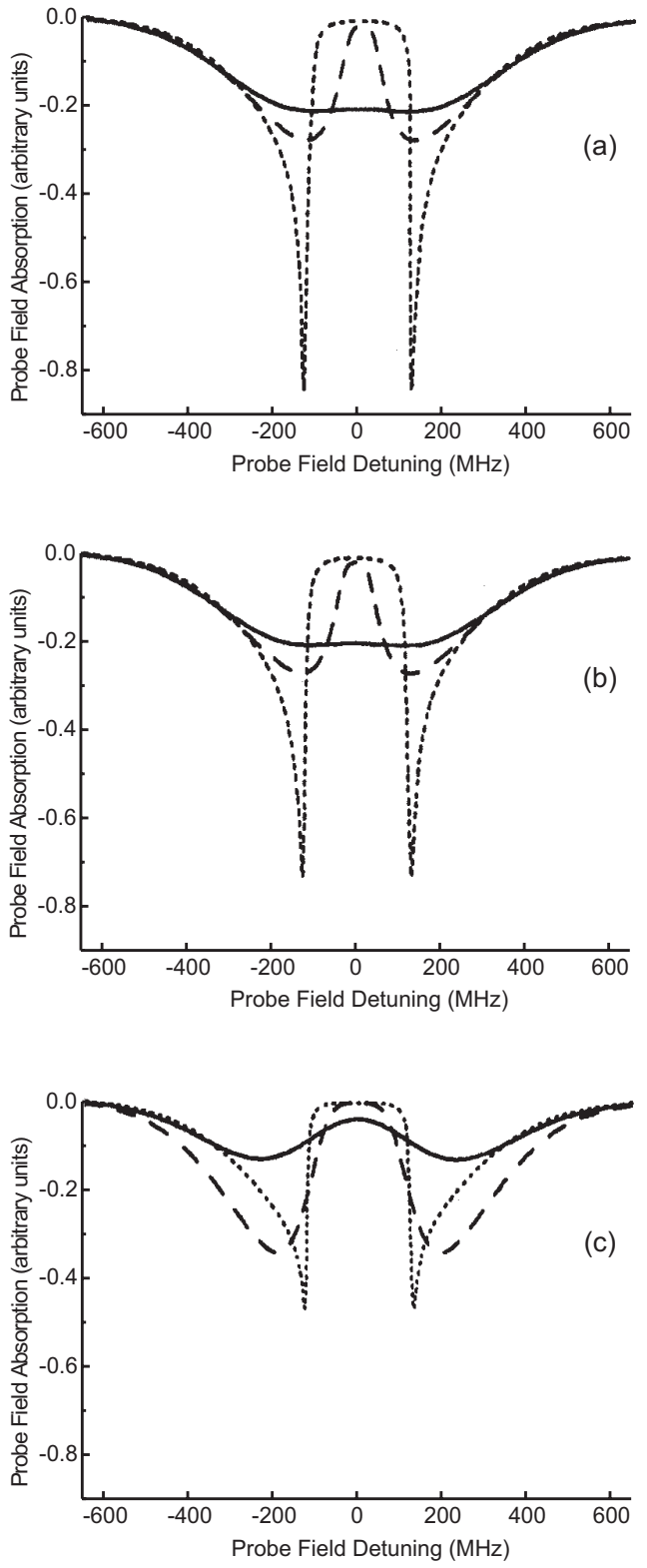

Fig. 11. Absorption of a weak probe field as a function of detuning for $\mathbf{a}$ ) the cascade, $\mathbf{b}$ ) the $\Lambda$, and $\mathbf{c}$ ) the V-type schemes. A resonant coupling field Rabi frequency $(=250 \mathrm{MHz})$ equal to half the Doppler width $(=500 \mathrm{MHz})$ is assumed present. In each case, three wavelengths regimes are considered: $\lambda_{\mathrm{c}}<\lambda_{\mathrm{p}}$ (dotted line), $\lambda_{\mathrm{c}}=\lambda_{\mathrm{p}}$ (dashed line), and $\lambda_{\mathrm{c}}>\lambda_{\mathrm{p}}$ (solid line). From Boon et al. (1999).

atoms, let us now proceed to explain how a somewhat analogous transparency can be attained in an ideal gas of two-level atoms irradiated by an intense, narrow-band laser beam tuned exactly to $\omega_{\mathrm{o}}$, the line center of the atomic transition. A strong, fully allowed, electric dipole transition is here assumed. Establishment of transparency in the spectral vicinity of $\omega_{0}$ would seem to be a fairly obvious requirement for a 2-level-atom LWI to operate in Space. As we will shortly see, in order for such a laser to develop sufficient optical gain via the pumping mechanism to be proposed, the 2-level-atom gas must also be 
(a)

(i)

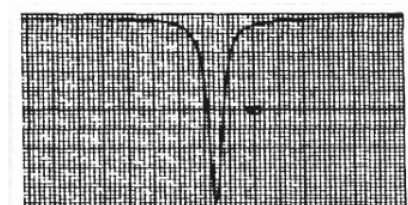

(ii)

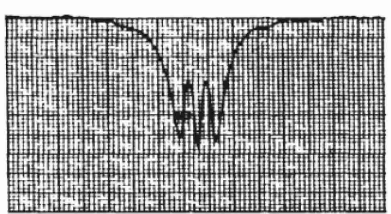

(iii)
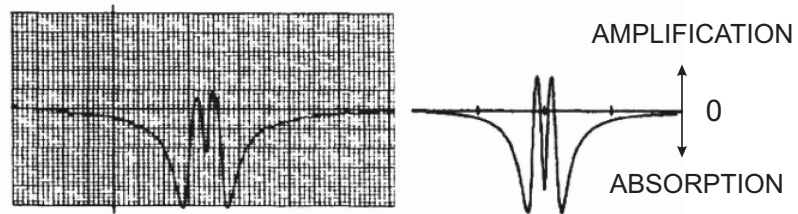

(iv)
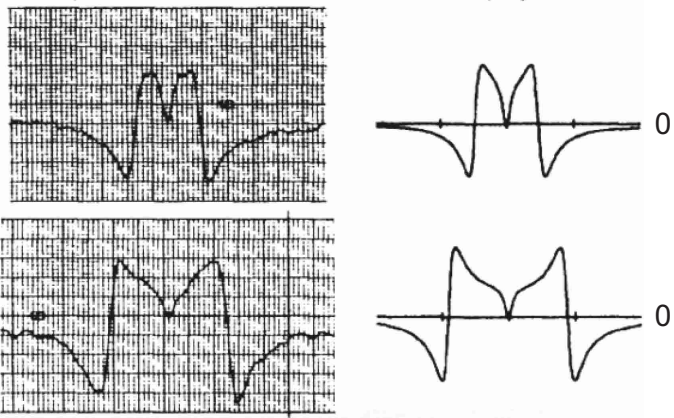

(v)
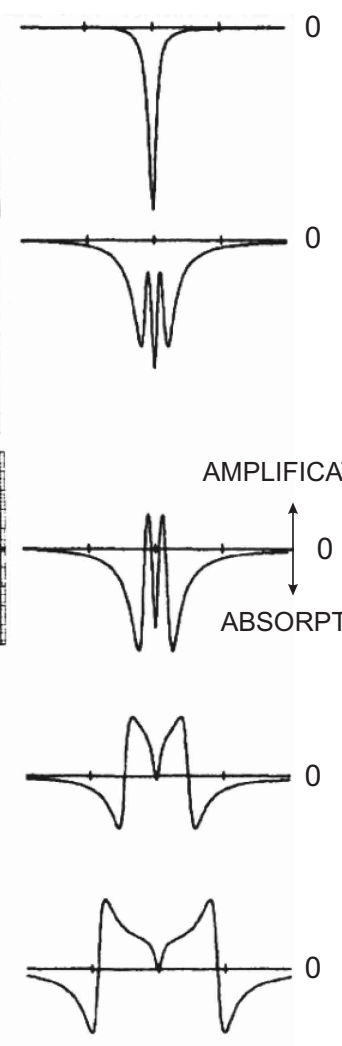

Fig. 12. a) Experimentally determined absorption-gain spectra (showing percent change of probe power) in a 2-level atomic gas (sodium) for various intensities of a continous-wave, monochromatic, resonant pump laser beam applied to the gas. Pump powers range from 0 (i) to $560 \mathrm{~mW} / \mathrm{cm}^{2}$ (v). Vertical scale per large division ranges from $1 \%$ (i) to $0.06 \%$ (v). b) Lineshapes calculated on the basis of the absorptiongain spectrum formula given in Mollow 1972. Horizontal scale $75 \mathrm{MHz} /$ div. From Wu et al. (1977).

irradiated by intense continuum radiation from a nearby illuminating star. It is thus assumed in the model that such radiation is present everywhere in a very broad spectral range that includes $\omega_{\mathrm{o}}$.

Figure 12 shows results from a landmark experiment (Wu et al. 1977) in which the absorption (gain) spectrum of a probe beam was measured for a gas of twolevel atoms (sodium) irradiated by an intense, monochromatic, continuous-wave beam tuned exactly to resonance. The experimental measurements are shown on the left. Computed spectra using the expression for the absorptionamplification line profile calculated by Mollow (Mollow 1972) are shown to the right. It is seen that very good agreement exists between theory and experiment. Note that as the power of the monochromatic resonant pump beam is increased from zero, the probe absorption spectrum takes on a distinctive three-lobed profile, with sidebands symmetrically displaced about the central frequency $\omega_{\mathrm{o}}$ by approximately $\pm \Omega$, where $\Omega$ is the Rabi frequency. As the pump beam power is increased still

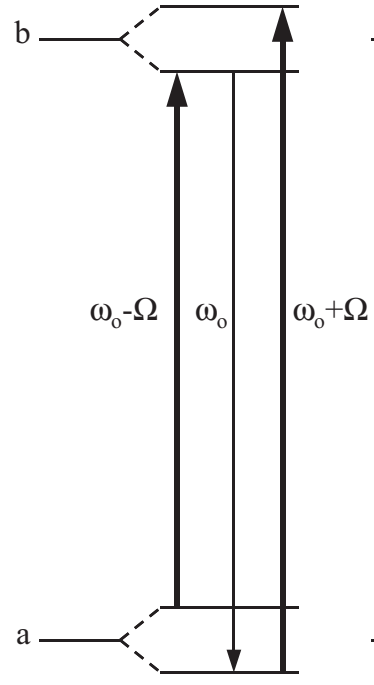

(a)

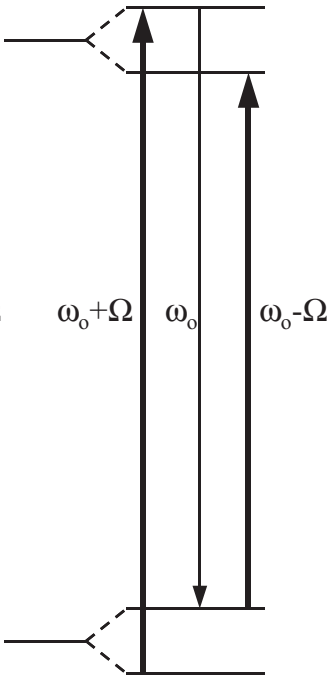

(b)
Fig. 13. a) and b) Diagrams showing the unit simultaneous three-photon processes involved in resonant SHRS pumping of two-level LWI's. The energy levels of a two-level atom are split as shown in the presence of a monochromatic field at $\omega_{\mathrm{o}} . \Omega$ is the Rabi frequency.

more, regions of gain appear between the outer absorption sidebands. All absorption and gain features become less intense as the laser power at $\omega_{\mathrm{o}}$ is increased. (The vertical scale in (i) is roughly twenty times larger than in (v).) At the highest pump power level shown, gain and absorption are seen to cancel exactly at the line center of the transition.

The spectra in Fig. 12 are usually explained in terms of the irradiated atom energy level diagram shown in Figs. 13a and 13b. When a monochromatic pump beam is applied exactly on resonance, the energy levels of a two-level atom are split as shown by the ac Stark effect (Autler \& Townes 1955). This leads to new resonance frequencies which can enhance various nonlinear processes, such as the simultaneous three-photon transitions indicated in the figure. Linear absorption processes occurring at the three allowed transition frequencies in Figs. 13a and $13 \mathrm{~b}$ can approximately account for the three-lobed absorption spectrum appearing at lower power levels of the monochromatic pump. As the pump power level is increased, both saturation (i.e. the tendency for the upper and lower electronic states to become equally populated at high pump powers) and quantum interference effects play important roles in determining the spectral shapes seen in Fig. 12. Saturation is largely responsible for the general decrease in intensity of all spectral features observed as the laser power at $\omega_{\mathrm{o}}$ is increased. Quantum interference accounts for the detailed spectral shapes and determines exactly which spectral regions show gain and which show loss. Without separating the exact contributions of each of these effects to the overall absorption-gain spectra shown in Fig. 12, one nonetheless can clearly see that application of an intense monochromatic resonant pump beam to a gas of 2-level atoms can by itself create the transparency 
conditions that would be needed in a space laser that operates at the same frequency. If a separate pumping mechanism could be found that would create photons at $\omega_{\mathrm{o}}$, one would then have the basis for an LWI that potentially could operate on a single allowed transition.

Consider first a situation in which there is only modest power at $\omega_{\mathrm{o}}$ present, so that the atom transition is not heavily saturated. In such a situation, it is reasonable to speculate that the resonant three-photon transitions depicted in Figs. 13a and 13b could satisfactorily serve to pump a 2-level-atom LWI. In the unit process for such transitions, two photons having frequencies that are both symmetrically disposed about $\omega_{0}$ and that fall within the outer absorption sideband profiles are simultaneously absorbed from the intense continuum irradiating the gas, a photon at $\omega_{\mathrm{o}}$ is created, and an atom is excited into the upper electronic state - with all four events occurring simultaneously. This nonlinear process is known as stimulated hyper-Raman scattering (SHRS). The SHRS transition probability per unit time is proportional both to the square of the continuum flux contained within the spectral width of the outer absorption sidebands, and to the total flux of the narrow-band laser beam itself. In a normal laser, a beam becomes amplified as it passes through a medium in which a population inversion exists. The production rate of new photons (i.e. the stimulated emission rate) is at any time proportional to the intensity present in the beam. With the SHRS pumping mechanism proposed here, the production rate of new photons is also proportional to the intensity of the beam being amplified, but no population inversion is involved. The resonant SHRS mechanism just described could only work for low intensities of the beam at $\omega_{0}$, since it requires that the population in the two upper Stark-split levels of Fig. 13 be less than that in the two lower Stark-split levels. It will be shown below that there is another resonant nonlinear process, four-wave mixing (FWM), that becomes the dominant gain-producing mechanism when the intensity of the beam being amplified (i.e. the beam at $\omega_{\mathrm{o}}$ ) becomes high.

The SHRS pumping process described above is fully resonant. Nonetheless, it is a nonlinear process. Nonlinear optical processes are practically unknown in astronomy. One should therefore now examine whether there is enough FUV continuum light in symbiotic stars to pump an LWI of the type here being considered, and whether such lasers can explain the superintense O VI (1032 , $1038 \AA$ ) doublet emissions that are observed. One can estimate how much a monochromatic beam at $\omega_{\mathrm{o}}$ would become amplified via SHRS in this space laser model by using the formula for the SHRS gain coefficient given as Eq. (5.22) in Hanna et al. (1979). This formula gives $\alpha_{\mathrm{I}}$, the intensity gain per unit length experienced by the monochromatic beam at $\omega_{\mathrm{o}}$, when a total pumping intensity $I_{\mathrm{c}}$ is applied at each of the Mollow absorption sidebands. The quantity $\alpha_{\text {I }}$ determines the amount of intensity amplification the beam receives exponentially, i.e. $I=I_{\mathrm{o}} e^{\alpha_{\mathrm{I}} \ell}$, where $\ell$ is the distance traveled by the light from $\ell=0$.
For simplicity, it is here assumed that the same transition dipole matrix element value $\mu$ holds for each of the allowed transitions in Figs. 13a and 13b. All energy differences appearing in the denominator of Eq. (5.22) in Hanna et al. (1979) are replaced by $\Gamma$, since all three participating waves are resonant. The SHRS gain thus becomes simply:

$\alpha_{\mathrm{I}}=g_{\mathrm{HR}} I_{\mathrm{c}}^{2} \simeq \frac{N \omega_{\mathrm{o}} I_{\mathrm{c}}^{2} \mu^{6}}{4 \varepsilon_{\mathrm{o}}^{3} c^{3} \hbar^{5} \Gamma^{5}}$.

Utilizing Eq. (4), one then has:

$\alpha_{\mathrm{I}}=g_{\mathrm{HR}} I_{\mathrm{c}}^{2} \simeq \frac{54 \pi^{3} N I_{\mathrm{c}}^{2} c^{6}}{\hbar^{2} \Gamma^{2} \omega_{\mathrm{o}}^{8}}$

To get an idea of the gain coefficient magnitude, we initially assume a two-level atom transition at $1000 \AA$, a value of $\Gamma$ equal to $5 \times 10^{8} \mathrm{rad} \mathrm{s}^{-1}$, a space atom density of $10^{4} \mathrm{~cm}^{-3}$, and a continuum pump power of $1 \mathrm{~W} / \mathrm{cm}^{2}$ in each of the two, $\simeq 160-\mathrm{MHz}$-wide, Mollow absorption sidebands. With these values substituted in Eq. (7), one finds the intensity gain coefficient of the beam at $\omega_{\mathrm{o}}$ to be $\alpha_{\mathrm{I}} \simeq 3 \times 10^{-8} / \mathrm{m}$.

To place this estimate of $\alpha_{\mathrm{I}}$ in the context of a typical symbiotic star environment, one must turn to measurements and estimates of the latter that have been made. For RW Hydrae, these were shown in Fig. 4. One presumes that the symbiotic star LWI is positioned close to the hot star surface. The light coherently generated by the LWI should radiate radially away from the white dwarf, perhaps preferentially towards the red giant, since it is material from the latter that accretes onto the hot white dwarf and causes it to be heated still more. Assuming the value of the hot star temperature $(180000 \mathrm{~K})$ estimated in Schild et al. (1996), one calculates from the blackbody photon distribution law (Allen 1976) that at $1000 \AA$, the blackbody continuum power emanating from the white dwarf surface is roughly $16.4 \mathrm{~W} / \mathrm{cm}^{2}$ in a $160 \mathrm{MHz}$ bandwidth. This implies that the $\alpha_{\mathrm{I}}$ value for an LWI in a typical symbiotic star environment would be about $8 \times 10^{-6} / \mathrm{m}$. With this value, one calculates that the beam at $\omega_{\mathrm{o}}$ would theoretically be amplified $\sim 10^{97}$ times in radially propagating away from the hot star surface a distance $\ell=0.04 \Re_{\odot}$ equal to the hot star radius. Thus, at least for low power levels of the narrow-band beam being amplified, the SHRS mechanism appears to provide enough optical gain for a symbiotic star LWI to operate.

For a given value of beam power, the two outer Mollow absorption bands are separated by twice the Rabi frequency, and one would expect that all continuum starlight overlapping these bands would be converted by SHRS into light in the beam at $\omega_{\mathrm{o}}$. Within the $\sim 160 \mathrm{MHz}$ spectral width of these bands, the continuum power radiated at the surface of the hot star in RW Hydrae is evidently something like $16.4 \mathrm{~W} / \mathrm{cm}^{2}$. As the power in the beam at $\omega_{\mathrm{o}}$ increases, the two Mollow absorption sidebands symmetrically move outwards, allowing continuum power in different spectral ranges to be converted into photons at $\omega_{\mathrm{o}}$. This behavior of the Mollow bands is illustrated in the 

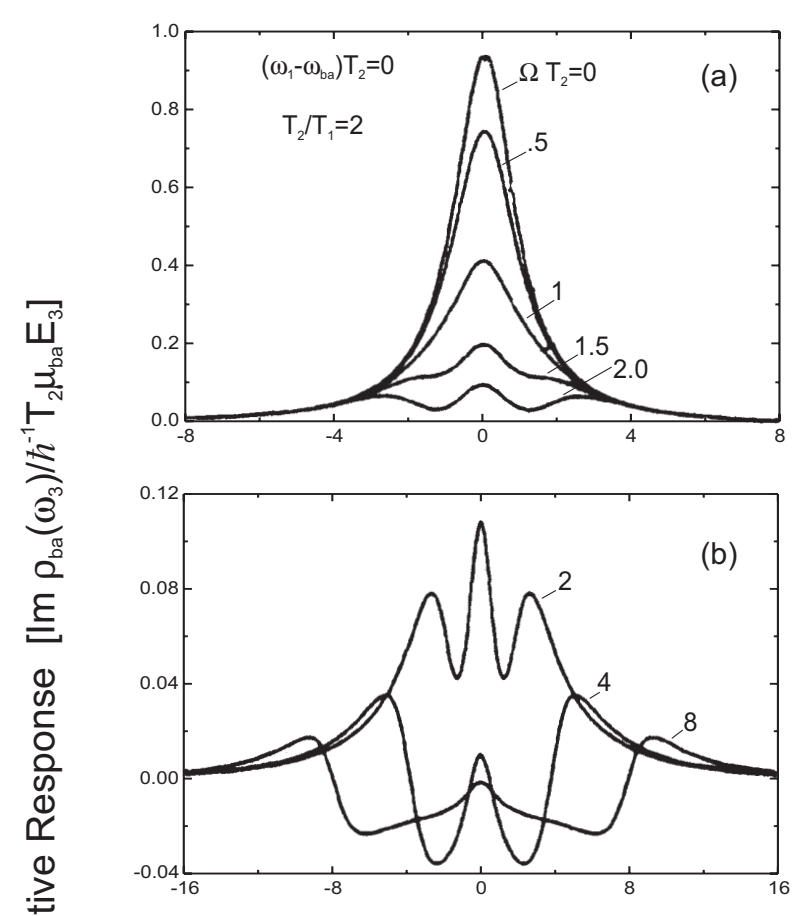

은

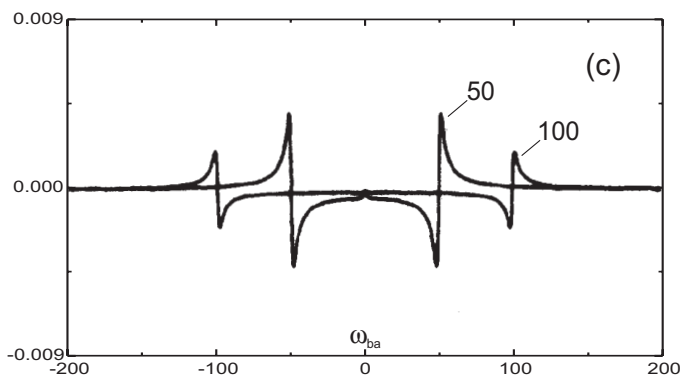

Probe - Wave Detuning $\left(\omega_{3}-\omega_{1}\right) T_{2}$

Fig. 14. Two-level-atom absorption spectrum as a function of probe detuning for a resonant monochromatic pump having a Rabi frequency $\Omega$. $T_{2}$ is twice the radiative decay time. From Fig. 2 of Boyd et al. (1981).

calculations shown in Fig. 14. One also again can see from this figure how rapidly the average strength of the absorption-gain features in the linear probe spectrum decreases with increasing power of the beam at $\omega_{0}-$ the result of saturation, as was indicated earlier. Saturation would also similarly prevent the above-postulated SHRS pumping process from occurring at high $\omega_{\mathrm{o}}$-beam intensities, and this now points to the possible existence of a severe logical inconsistency in our space laser model via the following argument (A. Kutyrev, private communication). Assume in Fig. 14 that $T_{1}=10^{-9} \mathrm{~s}$. Then, for the $\left(\Omega T_{2}=100\right)$ curve shown, the Rabi frequency is about $0.27 \mathrm{~cm}^{-1}$. Assuming that this curve corresponds approximately to the limit on the SHRS pumping process imposed by saturation, one can say that the maximum power that could be generated in the beam at $\omega_{\mathrm{o}}$ near the surface of the hot white dwarf in a symbiotic star would be about $1.6 \mathrm{~kW} \mathrm{~cm}^{-2}$. Next, consider any of the spectra shown in

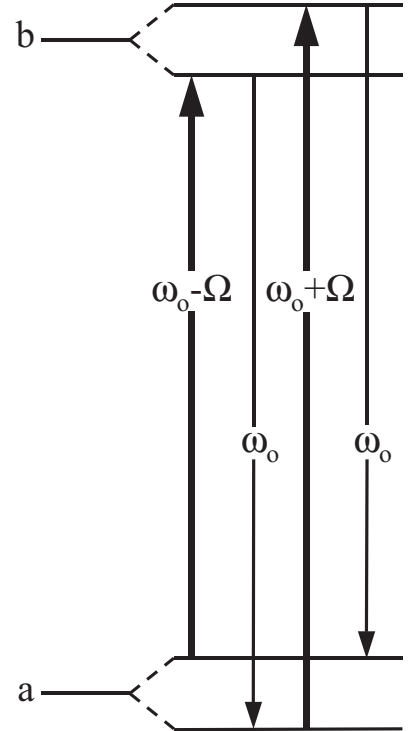

Fig. 15. Resonant four-wave mixing (FWM) process that creates photons at $\omega_{\mathrm{o}}$ at high values of $\Omega T_{2}$.

Figs. 6-8. These spectra were obtained with an instrument (the Berkeley spectrometer) for which the spectral resolution was $\sim 3000$. Consequently, each detector element (i.e. "bin") tallied all incident photons with frequencies falling within the $\sim 33 \mathrm{~cm}^{-1}$-wide spectral range monitored by the bin. For all bins, the incident photons included those representing light from the star's blackbody continuum. For one or possibly two of the bins, photons from the symbiotic star space laser beam at $\omega_{\mathrm{o}}$ would also have been included. However, whereas the star's continuum power recorded in any bin near $1000 \AA$ would have been about $101.5 \mathrm{~kW} \mathrm{~cm}^{-2}$, the upper limit of the power in the beam at $\omega_{\mathrm{o}}$ was estimated above to be $1.6 \mathrm{~kW} \mathrm{~cm}^{-2}$. Under such circumstances, it would thus be impossible to observe intense O VI FUV doublet emission lines from symbiotic stars.

The above difficulty is resolved if it can be shown that there is a new pumping mechanism which becomes effective when the Rabi frequency of the beam at $\omega_{\mathrm{o}}$ becomes very high, and that the new mechanism is capable of converting continuum starlight spanning a very wide frequency range into light at $\omega_{\mathrm{o}}$. The new mechanism here proposed is the resonant four-wave mixing (FWM) process diagrammed in Fig. 15. Since this mechanism is a multi-photon parametric process (i.e. it begins and ends on the same level), it is not as strongly diminished in intensity as SHRS when saturation occurs. Thus, it still remains effective in converting continuum light from the star into light in the beam at $\omega_{\mathrm{o}}$, even at very high intensities of the latter. If, for example, the FWM LWI pumping mechanism in a symbiotic star were capable of converting continuum starlight over a range $\omega_{\mathrm{o}} \pm 1500 \mathrm{~cm}^{-1}$ into monochromatic light at $\omega_{\mathrm{o}}$, the Berkeley spectrometer aboard the ORFEUS missions would have recorded symbiotic star O VI emission lines rising $\sim 90$ times above the star's continuum background, resulting in recorded 
spectra not totally dissimilar to those shown in Figs. 6-8. In this case, the power in the symbiotic star space laser beam near the surface of the hot white dwarf would be more than $9 \mathrm{MW} \mathrm{cm}^{-2}$. Thus, the FWM process potentially can capture symbiotic star continuum light over an enormous spectral range and turn it into narrow-band, coherent, laser light.

However, there is an essential feature that the proposed FWM pumping process must possess in order to be considered a viable mechanism. There exists a directly competitive FWM process that results in loss of photons at $\omega_{\mathrm{o}}$. This competitive process is the one obtained by reversing the directions of all arrows in the multi-photon scheme shown in Fig. 15. Since the maximum absolute values of the nonlinear cross-sections for both processes should be the same, it would seem that, on the average, photons at $\omega_{\mathrm{o}}$ would be neither gained nor lost. One is thus required to make an additional assumption regarding these two FWM processes. It is therefore here conjectured that if one were to compute a set of parameterized curves (the beam intensity at $\omega_{\mathrm{o}}$ being the parameter) plotting the nonlinear cross-section for gain at $\omega_{\mathrm{o}}$ (positive values indicating that photons at $\omega_{\mathrm{o}}$ are added; negative values indicating that photons are lost) as a function of frequency offset $\nu=\omega-\omega_{\mathrm{o}}$, with monochromatic light of intensity $I_{\mathrm{c}}$ being assumed present at both $\omega_{\mathrm{o}} \pm \nu$, the two outer portions of each curve would appear much as in Fig. 14c, that is, with positive values occurring at the largest frequency offsets from $\omega_{\mathrm{o}}$. Such a feature being present would always allow the FWM process that creates photons at $\omega_{\mathrm{o}}$ to deplete entirely the continuum photon density in any spectral range before the competing FWM process is able to interact with it. In the Appendix, arguments are presented to show why we believe the above conjecture to be true.

We now consider here briefly what might be happening when superintense $\mathrm{H}(\alpha)$ emission is seen in reddened, early-type stars. While the environments of symbiotic stars are well characterized, not much information exists about the environments of stars emitting superintense $\mathrm{H}(\alpha)$ radiation. Nonetheless, we outline for consideration a possible model for this emission by stating, and then briefly discussing, the two most essential features of this model. (1) Superintense $\mathrm{H}(\alpha)$ emission results from 2level lasing without inversion on the $\mathrm{H}(\alpha)$ transition. Twolevel, rather than 3-level, lasing without inversion must be presumed, because we know of no evidence showing Ly- $\beta$ emission in such stars. (2) The necessary H-atom population in the $2 \mathrm{~s}$ level is achieved via linear spontaneous resonance Raman scattering of light around Ly- $\beta$, as discussed in Sect. 2. It is presumed that a relatively dense cloud containing $\mathrm{H}$ atoms (and dust) engulfs the star without there being a significant H II region present. Significant population in the $2 \mathrm{~s}$ level results from the spontaneous Raman scattering rate of $\mathrm{H}$ atoms close to the star being comparable to $\Gamma_{21}$. When a star's continuum light near $6600 \AA$ is not enough to pump an $\mathrm{H}(\alpha)$ LWI, one sees $\mathrm{H}(\alpha)$ in absorption, as in Fig. 1a. While the continuum flux at $\sim 6600 \AA$ near the surface of an $\mathrm{H}(\alpha)$ star will be much less than that emitted near $1000 \AA$ at the surface of the hot white dwarf in a symbiotic star, a factor more than 3 million is gained in the value for $\alpha_{\mathrm{I}}$, due to the quantity $\omega_{\mathrm{o}}^{8}$ appearing in the denominator of Eq. (7).

\section{Conclusions}

It has here been proposed and argued that the superintense, narrow-band, (1032 $\AA, 1038 \AA$ ) O VI emission lines, which dominate the far-UV spectra of symbiotic stars, result from two-level lasing without inversion occurring in a gas of O VI ions located close to the surface of the hot white dwarf star in such space objects. The narrow-band stimulated emission light that is generated originates at the hot star surface, and then propagates radially outward from this surface in roughly spherical fashion, rapidly increasing in intensity as continuum light from the hot star becomes converted into narrow-band laser light by two nonlinear pumping mechanisms, stimulated hyper-Raman scattering (SHRS) and four-wave mixing (FWM). Closest to the star surface, where the narrow-band laser intensity initially starts to grow, SHRS is the dominant pumping mechanism. At greater distances from the star surface, where the laser power has greatly increased, FWM becomes the most important process. The FWM pumping mechanism in symbiotic stars appears to be a very efficient one, mainly because it sweeps into resonance with all spectral regions of the hot star's continuum light before a competing FWM process - one which would destroy photons at $\omega_{\mathrm{o}}-$ can do so. Via the FWM pumping mechanism, broadband continuum light from the hot star, contained within a spectral range extending symmetrically for perhaps a thousand or so wavenumbers on either side of the resonance, becomes entirely converted into narrow-band laser light. For typical symbiotic stars, the intensity of the narrow-band laser light in the vicinity of the hot star surface could easily attain values as high as $10 \mathrm{MW} / \mathrm{cm}^{2}$. Although the astrophysical environments of reddened, early-type stars are not as well known as those of symbiotic stars, it is suggested that when superintense $\mathrm{H}(\alpha)$ emission is seen in space objects of the former type, it too is the result of two-level-atom LWI.

Acknowledgements. We are grateful to the reviewer of the original manuscript we submitted on this subject for the suggestion that we quantify the main ideas that were proposed in that submission. We have especially benefited from discussions with D. J. Gauthier, B. D. Guenther, and J. E. Thomas of Duke University, and with A. Kutyrev, M. M. Freund, and T. R. Gull of the NASA GSFC.

\section{Appendix A: Theoretical treatment of FWM processes in irradiated 2-level-atom gases}

In this Appendix, evidence is presented to support the speculation made in Sect. 5 that, at high powers of the beam at $\omega_{\mathrm{o}}$, the maximum cross-sections for the FWM 
process shown in Fig. 15 occur at frequencies symmetrically offset from $\omega_{\mathrm{o}}$ by larger amounts than the frequencies at which the competitive FWM process maximizes. This evidence is based upon formulae contained in Boyd et al. (1981). These authors analyze four-wave parametric processes in strongly driven two-level systems, following an approach that is based upon density matrix techniques. (For ease in making numerical estimates based upon the formulae in Boyd et al. (1981), the Gaussian system of units employed by these authors is here utilized.)

In Sect. III of Boyd et al. (1981), the spatial propagation of two waves (at frequencies $\omega_{3}, \omega_{4}=2 \omega_{0}-\omega_{3}$ ) copropagating with an intense wave at $\omega_{\mathrm{o}}$ is studied. The waves at $\omega_{3}, \omega_{4}$ are assumed to be of weak intensity, while the intensity of the wave at $\omega_{\mathrm{o}}$ can be arbitrarily large. The form of the coupling assumed between the weak fields is expressed by writing the polarization amplitudes in terms of the susceptibilities as:

$$
\begin{aligned}
P_{3}= & \chi^{(1)}\left(\omega_{3},\left|E_{\mathrm{o}}\right|\right) E_{3} \\
& +\chi^{(3)}\left(\omega_{3}=2 \omega_{\mathrm{o}}-\omega_{4},\left|E_{\mathrm{o}}\right|\right) E_{\mathrm{o}}^{2} E_{4}^{*}, \\
P_{4}= & \chi^{(1)}\left(\omega_{4},\left|E_{\mathrm{o}}\right|\right) E_{4} \\
& +\chi^{(3)}\left(\omega_{4}=2 \omega_{\mathrm{o}}-\omega_{3},\left|E_{\mathrm{o}}\right|\right) E_{\mathrm{o}}^{2} E_{3}^{*},
\end{aligned}
$$

with the susceptibility elements being related to the elements of the density matrix by Eqs. (20a-d) of Boyd et al. (1981).

The above polarization amplitudes are then used as the source terms in the Helmholtz equations for the amplitudes of the waves at $\omega_{3}$ and $\omega_{4}$. Under the slowly varying envelope approximation, and for zero propagation vector mismatch $(\Delta k=0)$, the Helmholtz equations become:

$$
\begin{aligned}
& \frac{\partial A_{3}}{\partial z}=-\alpha_{3} A_{3}+\kappa_{3} A_{4}^{*}, \\
& \frac{\partial A_{4}^{*}}{\partial z}=-\alpha_{4} A_{4}^{*}+\kappa_{4}^{*} A_{3},
\end{aligned}
$$

where the coupling constants are given in terms of $\chi^{(1)}$, $\chi^{(3)}$ by Eqs. (24a-d) of Boyd et al. (1981).

The coupled amplitude Eqs. (A.3) and (A.4) can be solved for arbitrary initial values $A_{3}^{0}$ and $A_{4}^{0}$ of the field amplitudes $A_{3}(z)$ and $A_{4}(z)$ at the boundary of the nonlinear medium. Each of the solutions for the amplitudes $A_{3}$ and $A_{4}^{*}$ (given in Eqs. $(25 \mathrm{a}, \mathrm{b})$ of Boyd et al. 1981) consists of two terms having exponential $z$ dependence, with gain coefficients $g_{ \pm}$given by:

$g_{ \pm}= \pm \frac{1}{2}\left[\left(\alpha_{3}-\alpha_{4}\right)^{2}+4 \kappa_{3} \kappa_{4}^{*}\right]^{\frac{1}{2}}-\frac{1}{2}\left(\alpha_{3}+\alpha_{4}\right)$.

Although Eq. (A.5) assumes a very complicated form when the coupling constants are written generally in terms of the off-diagonal density-matrix elements $\rho_{\mathrm{ab}}$ (the latter given in Eqs. (5a)-(6), and (7) of Boyd et al. 1981), the situation becomes greatly simplified when the Rabi frequency $\Omega$ is much greater than $T_{2}^{-1}$, and when one assumes that $\omega_{3}, \omega_{4}$ are both located spectrally close to $\omega_{\mathrm{o}} \pm \Omega$. For simplicity, we also here assume a collisionless medium, so that $T_{2}=2 T_{1}$. One then finds, for example, that:

$$
\begin{aligned}
& \rho_{\mathrm{ba}}\left(\omega_{3}\right) \approx \frac{i \mu_{\mathrm{ba}} E_{3}\left(\rho_{\mathrm{aa}}-\rho_{\mathrm{bb}}\right)^{0}}{\hbar T_{2}\left[\nu^{2}-\Omega^{2}\right]} \\
& \rho_{\mathrm{ba}}\left(2 \omega_{\mathrm{o}}-\omega_{3}\right) \approx \frac{-i \mu_{\mathrm{ba}} E_{3}^{*}\left(\rho_{\mathrm{aa}}-\rho_{\mathrm{bb}}\right)^{0}}{\hbar T_{2}\left[\nu^{2}-\Omega^{2}\right]},
\end{aligned}
$$

where $\nu=\omega_{3}-\omega_{\mathrm{o}}$. From the above equations, and Eqs. (20a), (20c), (24a), and (24c) of Boyd et al. (1981), one finds that $\kappa_{3} / \omega_{3} \approx \alpha_{4} / \omega_{4}$. From an equivalent set of equations, one sees that $\kappa_{4} / \omega_{4} \approx \alpha_{3} / \omega_{3}$. From Eq. (A.5), one therefore deduces that $g_{+} \approx 0$ and that $\mathrm{g}_{-} \approx-\left(\alpha_{3}+\right.$ $\left.\alpha_{4}\right)$. Thus, when $\omega_{3}$ and $\omega_{4}=2 \omega_{\mathrm{o}}-\omega_{3}$ are in the vicinity of $\omega_{\mathrm{o}} \pm \Omega$, both waves grow exponentially with an intensity gain coefficient equal to:

$2 g_{-} \approx-\frac{8 \pi N \omega_{\mathrm{o}}\left|\mu_{\mathrm{ab}}\right|^{2}\left(\rho_{\mathrm{aa}}-\rho_{\mathrm{bb}}\right)^{0}}{c \hbar T_{2}\left[\nu^{2}-\Omega^{2}\right]}$.

From Eq. (A.8), one sees that positive gain exists for the FWM process that is competitive to the one shown in Fig. 15 only when $|\nu|<\Omega$. Only the FWM process of Fig. 15 occurs when $|\nu|>\Omega$. For large $\Omega$ values, the rate at which photons are added to the beam at $\omega_{\text {o }}$ via conversion of continuum photons in the two spectral intervals of width $\Delta \nu$, spectrally centered at $\omega_{\mathrm{o}} \pm \nu$, is given by:

$\frac{\mathrm{d} I_{\mathrm{o}}}{\mathrm{d} t}=4 c I_{(\mathrm{c}, \Delta \nu)} g_{-}$.

The expression in Eq. (A.9) has to be integrated over all values of $\nu$ greater than $\Omega$. The total rate of power transfer from the hot star continuum to the beam at $\omega_{\mathrm{o}}$ is thus approximately:

$$
\begin{aligned}
\frac{\mathrm{d} I_{\mathrm{o}}}{\mathrm{d} t}=\frac{16 \pi N \omega_{\mathrm{o}}\left|\mu_{\mathrm{ab}}\right|^{2}\left(\rho_{\mathrm{aa}}-\rho_{\mathrm{bb}}\right)^{0} I_{(\mathrm{c}, \Delta \nu)}}{\hbar T_{2}} \\
\times \int_{\Omega+T_{2}^{-1}}^{\infty} \frac{\mathrm{d} \nu}{\nu^{2}-\Omega^{2}}
\end{aligned}
$$

Since the integral in Eq. (A.10) equals $\frac{1}{2 \Omega} \log \left\{2 \Omega T_{2}\right\}$, the FWM power transfer rate is seen to fall off somewhat less rapidly than $\Omega^{-1}$.

One can start to apply Eq. (A.10) to O VI emission in symbiotic stars with substitution of the following values: $N=10^{4} \mathrm{~cm}^{-3}, \omega_{\mathrm{o}}=1.9 \times 10^{16} \mathrm{rad} \mathrm{s}{ }^{-1}$, $\mu_{\mathrm{ab}}=4.6 \times 10^{-18} \mathrm{esu}$, and $T_{2}=2 \times 10^{-9} \mathrm{~s}$. At a Rabi frequency of one wavenumber (i.e. $\Omega=2 \pi \times 3 \times 10^{10} \mathrm{rad} \mathrm{s}^{-1}$ ), one finds $\mathrm{d} I_{\mathrm{o}} / \mathrm{d} t \simeq 25 I_{(\mathrm{c}, \Delta \nu)}$. From Sect. 5 , the estimate for $I_{(\mathrm{c}, \Delta \nu)}$ was about $3 \mathrm{~kW} / \mathrm{cm}^{2}$ in a spectral bandwidth of one wavenumber. Thus, at a Rabi frequency of one wavenumber, the power in the beam at $\omega_{\mathrm{o}}$ is calculated to increase only at a rate $\simeq 75 \mathrm{~kW} \mathrm{~cm}^{-2} \mathrm{~s}^{-1}$. Since, in one second, the beam would have traveled a distance roughly equal to ten times the radius of the hot white dwarf, this rate of power transfer from the continuum to the beam at $\omega_{\mathrm{o}}$ is at least two orders of magnitude lower than what has been assumed throughout the present paper. The transfer 
rate calculated from Eq. (A.10) would roughly equal the number earlier assumed if the O VI ion density near the hot white dwarf were $\sim 10^{6} \mathrm{~cm}^{-3}$.

\section{References}

Allen, C. W. 1976, Astrophysical Quantities, 3rd ed. (Athlone Press, London and Dover, New Hampshire)

Autler, S. H., \& Townes, C. H. 1955, Phys. Rev., 100, 703

Boon, J. R., Zekou, E., McGloin, D., et al. 1999, Phys. Rev. A, 59, 4675

Boyd, R. W., Raymer, M. G., Narum, P., et al. 1981, Phys. Rev. A, 24, 411

Cohen, M., Anderson, C. M., Cowley, A., et al. 1975, ApJ, 196, 179

Gray, H. R., Whitley, R. M., \& Stroud, C. R. 1978, Opt. Lett., 3,218

Hanna, D. C., Yuratich, M. A., \& Cotter, D. 1979, Nonlinear Optics of Free Atoms and Molecules (Springer-Verlag, Berlin, Heidelberg, and New York)

Harris, S. E., Field, J. E., \& Imamoglu, A. 1990, Phys. Rev. Lett., 64, 1107

Harris, S. E., Field, J. E., \& Kasapi, A. 1992, Phys. Rev. A, 46, R29
Hau, L. V., Harris, S. E., Dutton, Z., et al. 1999, Nature, 397, 594

Kasapi, A., Jain Maneesh, Yin, G. Y., et al. 1995, Phys. Rev. Lett., 74, 2447

Liu, C., Dutton, Z., Behroozi, C. H., et al. 2001, Nature, 409, 490

Loudon, R. 2000, The Quantum Theory of Light, 3rd ed. (Oxford Univ. Press, Oxford)

Mollow, B. R. 1969, Phys. Rev., 188, 1969

Mollow, B. R. 1972, Phys. Rev. A, 5, 2217

Phillips, D. F., Fleischauer, A., Mair, A., et al. 2001, Phys. Rev. Lett., 86, 783

Rawlings, M. G., Adamson, A. J., \& Whittet, D. C. B. 2000, ApJS, 131, 531

Schild, H., Mürset, U., \& Schmutz, W. 1996, A\&A, 306, 477

Schmid, H. M. 1989, A\&A, 211, L31

Schmid, H. M., Krautter, J., Appenzellar, I., et al. 1999, A\&A, 348, 950

Stephenson, C. B. 1992, AJ, 103, 263

Weisskopf, V. 1931, Ann. Phys. Leipzig, 9, 23

Wu, F. Y., Ezekiel, S., Ducloy, M., et al. 1977, Phys. Rev. Lett., 38, 1077 